\title{
The underwater bubbles image's acquisition and processing based on Binocular Stereo Vision
}

\author{
Yu Guishui ${ }^{1, a}$, Liu Tao ${ }^{2, b}$ \\ ${ }^{1}$ Navy University of Engineer, Wuhan, 430033, China \\ ${ }^{2}$ Navy University of Engineer, Wuhan, 430033, China \\ altbebeto2002@163.com, bwangjaalex@163.com
}

Keywords: binocular stereo vision, mean Filter, median filter, sub-pixel edge detection

\begin{abstract}
Binocular Stereo Vision was used to observer and measure the underwater bububles. There are several ways have been taken to solve many difficulties in the process of the acquisition to get the image. Such as the equipment selection, the light sources selection, the approach to images and the feature extraction of the image. The most important of them is the noise elimination and the extraction of the bubbles. The feature of the image can be very obviously after the Median filter and Sub-pixel edge detection. In this way, it private an effective and feasible method to measure the underwater bubbles.
\end{abstract}

\section{Introductions}

The methods to measure the 3D data are validly used in many projects[1]. Especially in the way of machine vision measurement, it's a mature technology. The data it takes can reach the industry standard used in manufacture. Because of the less use in the underwater and the impact of the water to the lightways, the stereo vision is difficult to used in the underwater .In case of this situation; a proper method was taken out to acquire the underwater bubbles after a serials experiment. By comparing to the different ways to get the image, it has proved this method is quit usefull. And it also can provide the basic data of the underwater image to be used to calculate the information of the object.

\section{The Binocular Stereo Vision Observation System}

We can't get the 3D information of an object from one image, because in one image it just the length and the width that we can get. The Binocular Stereo Vision[2,3] can get the depth information by Triangulation with the use of Parallax theory.Figure1 is the schematic.

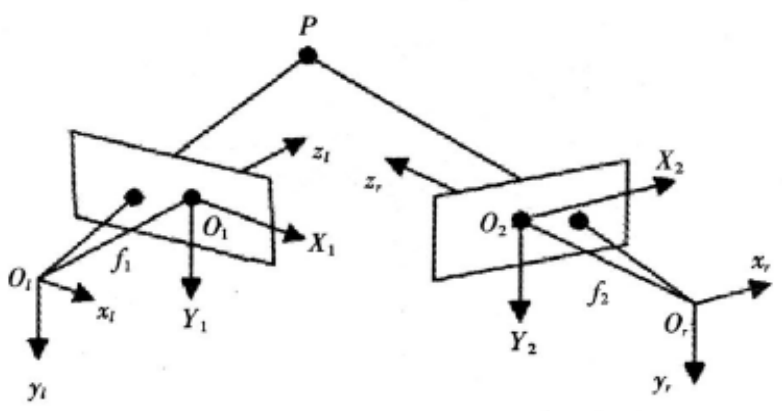

Fig.1 The principle of binocular stereo vision model

The 3D coordinates can be calculated by the 2D coordinates with the use of this model above[4].

With the camera perspective transformation model can get 


$$
S_{r}\left[\begin{array}{c}
X_{r} \\
Y_{r} \\
1
\end{array}\right]=\left[\begin{array}{ccc}
f_{r} & 0 & 0 \\
0 & f_{r} & 0 \\
0 & 0 & 1
\end{array}\right]\left[\begin{array}{c}
x_{r} \\
y_{r} \\
z_{r}
\end{array}\right],
$$

with this formula can calculate the relationship between the two cameras.

$$
\rho_{r}\left[\begin{array}{c}
X_{r} \\
Y_{r} \\
1
\end{array}\right]=\left[\begin{array}{cccc}
f_{r} r_{1} & f_{r} r_{2} & f_{r} r_{3} & f_{r} t_{x} \\
f_{r} r_{4} & f_{r} r_{5} & f_{r} r_{6} & f_{r} t_{y} \\
r_{7} & r_{8} & r_{9} & t_{z}
\end{array}\right]\left[\begin{array}{c}
z X_{1} / f_{1} \\
z Y_{1} / f_{1} \\
z \\
1
\end{array}\right],
$$

Hence the 3D coordinate of the point can described as

$$
\left\{\begin{array}{l}
x=z X_{1} / f_{1} \\
y=z Y_{1} / f_{1} \\
z=\frac{f_{1}\left(f_{r} t_{x}-X_{r} t_{z}\right)}{X_{r}\left(r_{7} X_{1}+r_{8} Y_{1}+f_{1} r_{9}\right)-f_{r}\left(r_{1} X_{1}+r_{2} Y_{1}+f_{1} r_{3}\right)}, \\
=\frac{f_{1}\left(f_{r} t_{y}-Y_{r} t_{z}\right)}{Y_{r}\left(r_{7} X_{1}+r_{8} Y_{1}+f_{1} r_{9}\right)-f_{r}\left(r_{4} X_{1}+r_{5} Y_{1}+f_{1} r_{6}\right)}
\end{array}\right.
$$

some parameters have given, the focus $f_{1}, f_{r}$ and the image coordinate system of the point. So we can get the 3D ordinates of the piont as soon as we get the ratation matrix $R$ and translation vector.

The images of the Binocular Stereo Vision are captured by two cameras in the same time at different places. We need a high quality CCD for the image feature of the underwater's object is too weak to get enough information for calculation. So what we need is the special CCDs which can take two photos and the same time strictly. In the experiment, we use the Micro view's MVC360SAC-GE60ST2 which is a small CCD system with two CCDs can take photos at the same time. Both CCD have the same resolution $(752 \times 480)$ and $60 \mathrm{fps}$ (with the low resolution can get 200), 8 Bits. The bubble comes from a small ceramic which has plenty of tiny holes on the surface. Using an air pump the airflow goes into the hole of the ceramic and come out as the bubble we want. We can get the floating bubbles which come from the ceramic at the bottom of the glass tank.

\section{The processing of the underwater bubbles}

When at the time one exposure happened, the bubble had moved a little length. But compared with the bubble' s size, the length is big enough to make the tail pattern on the image[5]. Basically the photo we get from one bubble is the move length of it at the time that one exposure of the camera happened. In order to eliminate the tail pattern we had to narrow the exposure time. So we can improve the image of the bubble by take a better camera which had a shorter exposure time.

In the Binocular Stereo Vision the image must be totally the same and the process to denoise is also the same. Only in this way can make sure the images have the same information and can reduce the error in the process of image matching[6]. We take one of the photo pair as example to explain the impact of the different image denoising.

The mean filter[4] is an instance of a linear filter. The basic view of the mean filter is that by using the average number of one point' s neighborhood to removal the mutation point. Through this step can denoise part of the noise. It can be done by the convolution which contained in the box template. The box template is that its entire coefficient has the same value. The common template is $3 \times 3$ and $5 \times 5$ as below 


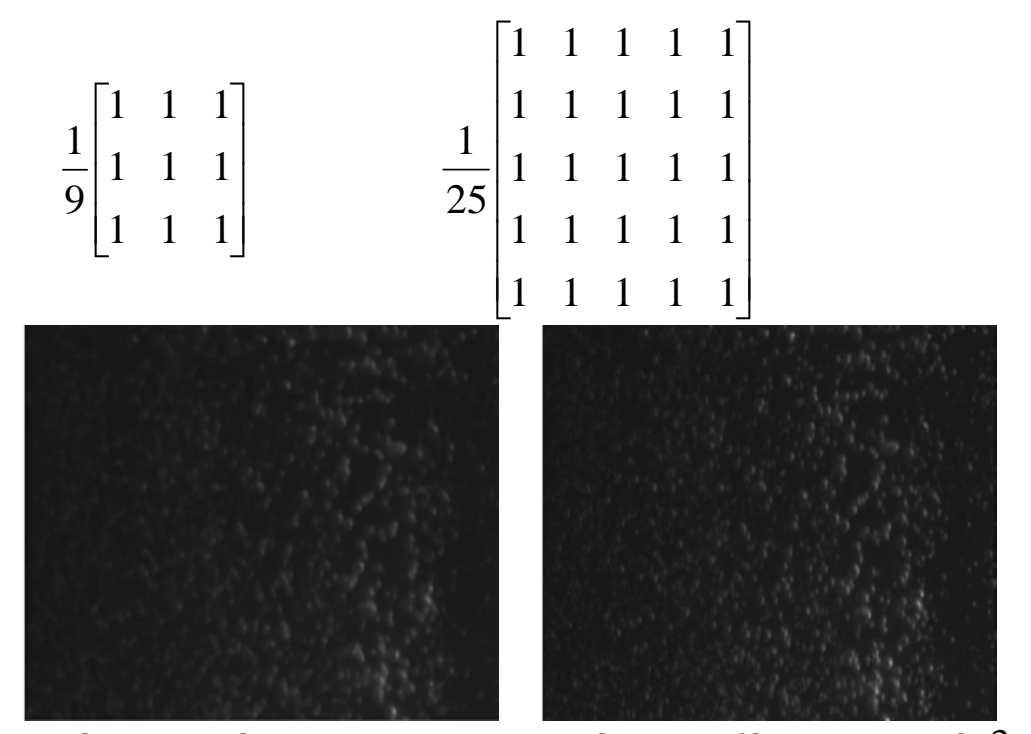

Fig.3 The original image

Fig.4 The mean filter image with $3 \times 3$ template

The Median Filter is an instance of a nonlinear. It sets a point's gray from the median of the field's all points. It also can describe as this that through a slide window the center of the window's value can be replaced by calculates the median value of all the points in the window. There is a one-dimensional sequence as $f_{1}, f_{2}, \cdots, f_{n}$, the length of the window is mother process of median filter is that take $f_{i-v}, \cdots, f_{i-1}, f_{i}, f_{i+1}, \cdots f_{i+v}$ one by one ( $f_{i}$ is the value of the window center), then sort them by size. The output is the median number of the sort results. It given as below:

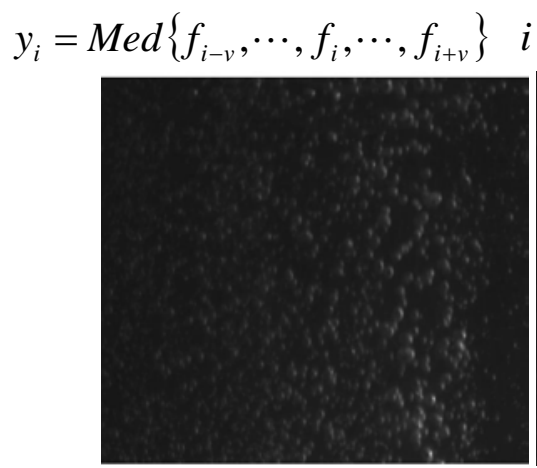

Fig.5 The original image

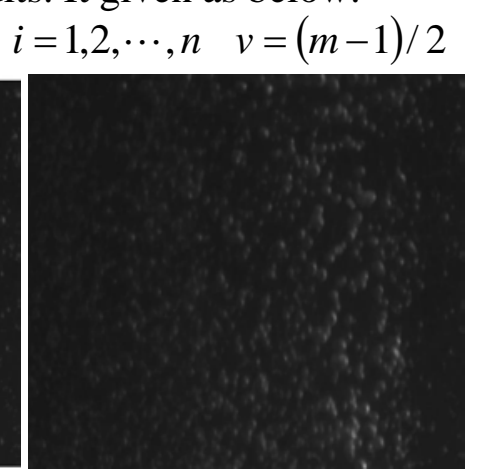

Fig.6 the median filter image with $3 \times 3$

It showed that the edges of the image are become fuzzy. Though its noise has been denoise a lot but the edges are not obvious for the latter edge detection. Compared with the histogram of the images above it's obvious that the $3 \times 3$ template median is the most effective for the underwater images that have low illumination.

After image denoise, we need to extract the bubble's profile in accuracy[7,8]. In the water there maybe some other tiny impurities, so we need to remove the interference for the extraction. And because the size of the bubble is very small, it's hard to detect the edges of it. So we take the sub-pixel edge detection as the main method.

The random pixel $\left(x_{0}, y_{0}\right)$ can be presented by its adjacent pixel in Taylor polynomial.

$$
\begin{aligned}
& f(x, y)=g\left(x_{0}, y_{0}\right)+\left(\left(x-x_{0}\right)\left(y-y_{0}\right)\right)\left(\begin{array}{l}
g_{x}\left(x_{0}, y_{0}\right) \\
g_{y}\left(x_{0}, y_{0}\right)
\end{array}\right) \\
& +\frac{1}{2}\left(\left(x-x_{0}\right)\left(y-y_{0}\right)\right)\left(\begin{array}{ll}
g_{x x}\left(x_{0}, y_{0}\right) & g_{x y}\left(x_{0}, y_{0}\right) \\
g_{x y}\left(x_{0}, y_{0}\right) & g_{y y}\left(x_{0}, y_{0}\right)
\end{array}\right)\left(\begin{array}{l}
\left(x-x_{0}\right) \\
\left(y-y_{0}\right)
\end{array}\right)
\end{aligned}
$$

$g_{x}, g_{y}, g_{x x}, g_{x y}, g_{y y}$ is the partial derivative from the convolution result of the image and the Gaussian kernel.

The figure below is the edge detective image. It showed that as the edge feature is not very obvious to detect it, not all the bubbles can be detected. Some can't be detected because the gray 
value of the bubble is similar to the background. From the histogram of the image we can find out the bimodality is very good. It also showed that only the bubbles which have the good illumination condition can be detected clearly. So we need the good light source to get the image good enough to be detected.

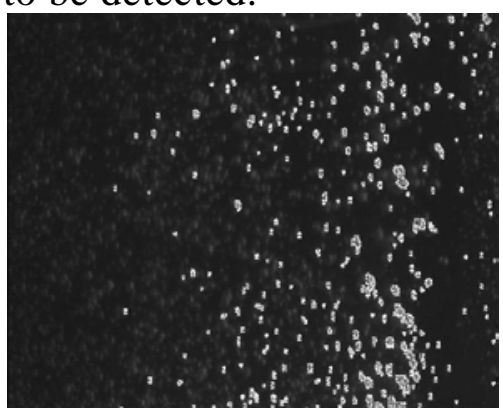

Fig.9 The detective image

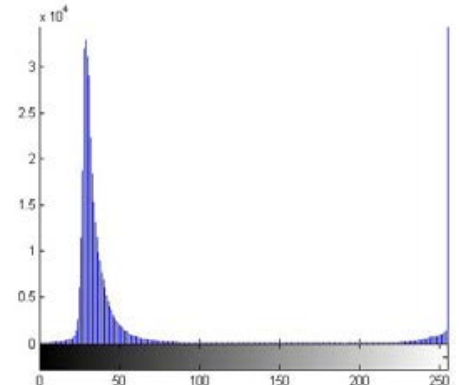

Fig.10 The Histogram of the detective image

\section{Summary}

By the experiment we have done above, we know that the most important technology is the light source. It's the base of the whole image quality. But there different ways of the light source to different objects underwater. We take the bubbles as the sighting vane. It's for the reason that the bubbles come out one after another as a whole so we can take the illumination in a scale. In order to decrease the index of refraction we chose the monochromatic light as the light source. The process of the image can validly used in the underwater background with low illumination, especially the image denoise and edge extraction. They have been proved by the experiment above.

The underwater observe is a techongy acquires a lot laboratory experience. It's very useful in the area of hydrodynamics, chemistry etc. We just give the option of the basic experiment and the effective process underwater. It needs a lot of work to do in the research to make it perfect.

\section{References}

[1] Xiao Xinyuan,Shi Yonghua,Wang Guo-rong.Underwater welding seam image acquisition and recognition based on binocular vision, manufacturing automation, 11(2008)90-92.

[2] Zhang Guangjun. Machine vision, Science Press, Beijing, 2008.

[3] R. Boyle and R. Thomas. Computer Vision: A First Course, Blackwell Scientific Publications, 1988, pp $32-34$.

[4] Sui Jing, Jin Weiqi. Implementation and progress of binocular stereo vision technology, the application of electronic technology, 17(1988)32-34.

[5] Carsten Steger. Machine Vision Algorithms and Applications, Science Press, 2004.

[6] Yan Ni. Research on the key technology of bubble behavior of gas-liquid two-phase flow field in 3D measurement, Tianjin University, 2006.

[7] Zhang Jianxin, Duan Fajie, Zhong Ming, Ye Shenghu. Study on Application of Binocular Sensor in3D Measurement, Journal of Measurement, 4(1999)96-100.

[8]Li Yi, Yang Wenyu. Modeling based on the system of double CCD stereo vision, Huazhong Univ.of Sci.\&Tech, 8(2004)5-7. 